$\underline{\text { Research article }}$

\title{
Diagnosis of toxoplasmic lymphadenopathy: comparison of serology and histology
}

\author{
WMDR Iddawela $^{1,} \mathrm{~K}_{\text {Ehambaram }}{ }^{1,}$ DRLN Bandara ${ }^{1}$ \\ Sri Lankan Journal of Infectious Diseases 2015 Vol.5 (1):2-6 \\ DOI: http://dx.doi.org/10.4038/sljid.v5i1.7746
}

\begin{abstract}
Introduction: Toxoplasma gondii is a zoonotic protozoan that infects most species of birds and mammals. It can cause neurologic or ocular disease with wide ranging manifestations. Human infection is usually asymptomatic, but some may develop fever, lymphadenopathy, malaise, chills, sweats, headaches or myalgia. Presence of lymphadenopathy is considered as a positive sign of toxoplasmosis. This is confirmed by biopsy of the inflamed lymph node.

Objective: The present study was carried out to determine the reliability of the histological findings in the diagnosis of toxoplasmosis.

Methods: Biopsy reports of seventeen patients who presented with lymphadenopathy were studied. ELISA was carried out in serum samples of the 17 individuals. Toxoplasma IgG avidity ELISA was performed to identify the phase of infection (acute/chronic). PCR was performed to confirm the results obtained by ELISA.

Results: Six cases were found to be seropositive. However all 17 cases had a positive biopsy report. PCR was performed to detect bands specific to $T$. gondii but they were absent. This result was supported by Toxoplasma IgG avidity testing which revealed that all six positive samples had high avidity thus suggesting that the infection was chronic.

Conclusion: The study revealed that histological findings produced $64.7 \%$ (11/17) false positive results. Chronic infection does not have the organism in the blood stream resulting in the absence of DNA for PCR amplification which in turn explains the absence of bands specific for T. gondii. Thus it is recommended that serology (ELISA) be used to diagnose toxoplasmosis.
\end{abstract}

Key Words : Toxoplasma gondii, diagnosis, lymphadenopathy, histology, ELISA, PCR.

${ }^{1}$ Department of Parasitology, Faculty of Medicine, University of Peradeniya

Address for correspondence: Dr Devika Iddawela, Department of Parasitology, Faculty of Medicine, University of Peradeniya, Sri Lanka Telephone:071-4460866 Email: devikaiddawela@yahoo.com

Received 9 December 2014 and revised version accepted 19 March 2015 


\section{Introduction}

Toxoplasma gondii is a widespread zoonotic protozoan that infects a wide range of birds, mammals and reptiles. Humans acquire infection by ingesting oocyst-contaminated food and water, during organ transplantation, blood transfusion, laboratory accidents, or congenitally. ${ }^{1}$ They can remain dormant within tissues for the life of the host. T. gondii human infection is found worldwide, in a variety of climates and socio-economic circumstances. Prevalence of toxoplasmosis can vary with age and geographical origin. ${ }^{2}$ Clinical presentation of $T$. gondii infection also depends on the age and immune status of the patient. ${ }^{3}$ It can cause severe neurologic or ocular disease in the foetus during pregnancy. Ocular diseases include retinochoroiditis and anterior uveitis among other manifestations. ${ }^{1}$ In the majority of patients who are immune-competent, primary infection is usually asymptomatic. ${ }^{3}$ However, some patients may develop fever, lymphadenopathy, malaise, chills, sweats, headaches or myalgias. The lymph nodes are usually discreet, non-tender, and do not suppurate. ${ }^{3}$ A distinct histological form of lymphadenitis observed in toxoplasmosis was described by Piringer-Kuchinka et al., in $1958 .{ }^{4}$ Diagnosis of toxoplasmic lymphadenopathy is important in immune-competent hosts to differentiate it from neoplasia. However the histological findings cannot be fully relied upon to treat the patients since lymphadenopathy is seen in many other conditions such as Epstein-Barr virus and other mononucleosis-like illnesses including cytomegalovirus and HIV with acute retroviral syndrome. The present study was therefore carried out to determine the reliability of histopathology in the diagnosis of toxoplasmosis.

\section{Materials and Methods}

\section{Subjects}

Seventeen subjects, with a histological suspicion of Toxoplasma lymphadenitis, referred for confirmation of the diagnosis of toxoplasmosis to the Department of Parasitology, Faculty of Medicine, University of Peradeniya, during a 2 year period (2012- 2013), were included in this study. Medical histories of all 17 patients, including location of lymphadenopathy, were obtained. Other details such as age and sex were collected. Biopsy reports of all 17 patients were reviewed.

Venous blood $(2 \mathrm{ml})$ was collected from each patient into individual blood collecting tubes and serum separated by centrifugation and stored at $-20^{\circ} \mathrm{C}$ until investigated.

Serum was examined for the presence of $T$. gondii specific $\operatorname{IgG}$ antibodies using an in-house Toxoplasma Excretory Secretory-ELISA developed by the method described by Meira et al. ${ }^{5}$ Toxoplasma IgG avidity testing was carried out on the Toxoplasma positive samples to determine if the infection was chronic or acute. PCR was carried out on all six blood samples collected from sero-positive cases.

\section{Toxoplasma IgG antibody assay}

Sera of all 17 patients were assayed for anti Toxoplasma IgG by the in-house ELISA. The flat bottomed ELISA plate was coated with somatic tachyzoite antigen at a concentration of $0.000846 \mu \mathrm{g} / \mathrm{ml}$ and incubated overnight at $4{ }^{\circ} \mathrm{C}$. Unbound antigen was removed by washing the 
plate with wash buffer (phosphate buffered saline, PBS, with $0.05 \%$ Tween 20) thrice and blocked with blocking buffer (PBS containing sucrose and bovine serum albumin) for an hour at room temperature. Excess blocking agents were removed by washing thrice with wash buffer. A 1:100 dilution of the patient sera was prepared in phosphate buffered saline (PBS), aliquoted to the wells and left to bind for an hour at room temperature. Washing was done thrice and the plates were incubated for a further 1 hour at room temperature with horse radish peroxidase conjugated anti-human IgG diluted 1:5000 in PBS. After another washing cycle, substrate was added and left for 20 minutes in the dark for the reaction to take place. Color development was stopped by adding $50 \mu \mathrm{l}$ of $2 \mathrm{M} \mathrm{H}_{2} \mathrm{SO}_{4}$ (sulphuric acid), and the absorbance measured using an ELISA reader (Erba Lisa Scan II) with a $492 \mathrm{~nm}$ filter. Positive and negative controls were set up simultaneously. Each serum sample was assayed in triplicate.

\section{Polymerase chain reaction}

DNA was isolated using commercial methods (Qiagen). PCR amplification of highly conserved repetitive gene B1of $T$. gondii was done using the forward primer, TG18S48_F, (5 -CCA TGC ATG TCT AAG TAT AAG C-3`) and reverse primer, TG18S359_R, (5`-GTT ACC CGT CAC TGC CAC-3). The PCR products were run on a $1.5 \%$ agarose gel at $100 \mathrm{~V}$ and $250 \mathrm{~mA}$ for 45 min. The gel was stained in ethidium bromide for $20 \mathrm{~min}$ and observed under UV light (302nm). Gel image was captured using the software Alpha Imager mini.

\section{Toxoplasma IgG avidity testing}

Avidity assay was performed according to commercial methods. ${ }^{6}$ Avidity was assessed by setting up triplicate sets of the routine Toxoplasma ELISA for T. gondii specific IgG. After initial incubation with antibody, one set was washed with $6 \mathrm{M}$ urea for $8 \mathrm{~min}$ and the other set was washed with wash buffer lacking urea. Absorbance value of the set washed with urea was divided by the value of the set washed with buffer lacking urea. This provided the avidity index (AI). AI values $<0.20$ indicate low avidity, values of $0.20-0.25$ indicate intermediate avidity, and values $>0.25$ indicate high avidity. ${ }^{6}$

\section{Results}

Age of the participants ranged between 3 and 68 (mean=26.5) years. The majority were males $(\mathrm{n}=10,58.8 \%)$. Most patients had cervical lymphadenopathy $(\mathrm{n}=15,88.2 \%)$, while only a small percentage had axillary $(n=1,5.9 \%)$ and inguinal lymphadenopathy $(n=1,5.9 \%)$. The biopsy reports issued by pathologists were reviewed and it was found that all the 17 patients were histologically positive for toxoplasmosis. Six of the 17 patients $(35.3 \%)$ with a positive biopsy report were sero-positive for toxoplasmosis. None of the serology positive patients were PCR positive. Toxoplasma IgG avidity testing revealed that all the 6 positive samples had high avidity, suggesting chronic infection.

\section{Discussion}

Prevalence of toxoplasmosis varies from 15 to $77 \%$ in different parts of the world, and between different population groups within the same country. ${ }^{7}$ Sri Lanka, India and Bangladesh have been categorized in the intermediate prevalence group (10-40\%) amongst Asian countries. ${ }^{8}$ 
Toxoplasmosis in the majority of people is asymptomatic. ${ }^{9}$ Lymphadenopathy is the most frequent clinical presentation of toxoplasmosis in immune-competent patients with 3-7\% prevalence. $^{10,11}$ In this study group, positive antibody carriage of only $35.3 \%$ was observed among patients histologically diagnosed as having Toxoplasma lymphadenopathy, indicating low reliability of histology in the diagnosis of toxoplasmic lymphadenopathy. Findings are comparable to a study done by Eapen et al., ${ }^{12}$ while some other studies have reported even lower figures of $22.5 \%$. $^{13}$

Diagnosis of toxoplasmosis can be done by a number of methods, either directly by PCR, hybridization, histology and isolation or via serological methods. Serological methods include ELISA, IgG avidity test, Sabin-Feldman dye test and immune-fluorescent antibody test. High avidity IgG rules out primary infections of less than five months. ${ }^{14}$ Serology based on demonstration of $T$. gondii specific antibodies is the most commonly used technique to diagnose toxoplasmosis. ${ }^{15}$ Absence of $\mathrm{IgG}$ antibodies clearly excludes Toxoplasma infection. In the present study, the majority of histologically suspected cases $(64.7 \%)$ were sero-negative indicating a high level of false positive results. Since toxoplasmic lympadenopathy has no specific clinical features, the diagnosis is usually carried out by histology or serology. Specific histological diagnosis is based on specific reactive changes in the lymph node and not on identifying the parasite. At low magnification, there is periadenitis with capsular inflammation and scarring. Follicles are large and irregular with active germinal centers containing many tingible body macrophages. Scattered collections of pale staining parafollicular monocytoid B cells and small clusters of epithelioid histiocytes forming small granulomas are the most distinct features of toxoplasmic lymphadenitis. It is extremely rare to identify toxoplasma cysts in lymph nodes. ${ }^{16}$ Even with an experienced pathologist, $100 \%$ diagnostic efficacy cannot be achieved. ${ }^{17}$ The present study identified a large proportion of false positivity with histological diagnosis, stressing the importance of confirmatory diagnosis using serology. Therefore, carrying out other diagnostic tests including serology is mandatory for definite diagnosis.

\section{Conclusion}

This study revealed comparatively low sensitivity of histological diagnosis of Toxoplasma lymphadenopathy, emphasizing the need for confirmatory serological analysis.

\section{Conflicts of Interest}

The authors have no conflicts of interest to declare.

\section{Acknowledgement}

We would the like to express our gratitude to National Research Council for funding (Grant 0738).

\section{References}

1. Elmore SA, Jones JL, Conrad PA, et al . Toxoplasma gondii: epidemiology, feline clinical aspects, and prevention. Trends Parasitol. 2010; 26(4):190-6. doi: 10.1016/j.pt.2010.01.009 
2. Ivanova K, Glatz K, Zippelius A, et al . Acute toxoplasmosis mimicking melanoma metastases: review of conditions causing false-positive results on (18)F-FDG PET/CT. Dermatology. 2012; 225(4):349-53. doi: 10.1159/000346333

3. Taila AK, Hingwe AS, Johnson LE. Toxoplasmosis in a patient who was immunecompetent: a case report. J Med Case Rep. 2011; 18:5-16. doi: 10.1186/1752-1947-5-16

4. Piringer-Kuchinka A, Martin I, Thalhammer O. Superior cervical nuchal lymphadenitis with small groups of epitheloid cell proliferation. Virchows Arch. 1958; 331(5):522-35. No doi

5. Meira CS, Costa-Silva TA, Vidal JE, et al. Use of the serum reactivity against Toxoplasma gondii excreted-secreted antigens in cerebral toxoplasmosis diagnosis in human immunodeficiency virus-infected patients. J Med Microbiol. 2008; 57:845-50. doi: 10.1099/jmm.0.47687-0

6. Toxoplasma Ig $\mathrm{G}$ avidity testing, Focus Diagnostics. Available at http://www.focusdx.com/focus/techsheets/ToxplasmaAvidity.pdf

7. Jones JL, Kruszon-Moran D, Wilson M, et al. Toxoplasma gondii infection in the United States: sero-prevalence and risk factors. Am J Epidemiol. 2001; 154(4):357-65. http://dx.doi.org/10.1093/aje/154.4.357

8. Yano Y, Nam HW, Abdullah KA, et al. Toxoplasmosis and Babesia in Asia. Asian Parasitology. 2000; 4:2-4. No doi

9. Hill DE, Chirukandoth S, Dubey JP. Biology and epidemiology of Toxoplasma gondii in man and animals. Anim Health Res Rev. 2005; 6(1):41-61. http://dx.doi.org/10.1079/AHR2005100

10. Dubey JP, Jones JL. Toxoplasma gondii infection in humans and animals in the United States. Int J Parasitol. 2008; 38(11):1257-78. doi: 10.1016/j.ijpara.2008.03.007

11. McCabe RE, Brooks RG, Dorfman RF, et al. Clinical spectrum in 107 cases of toxoplasmic lymphadenopathy. Rev Infect Dis. 1987; 9(4):754-74. http://dx.doi.org/10.1093/clinids/9.4.754

12. Eapen M, Mathew CF, Aravindan KP. Evidence based criteria for the histopathological diagnosis of toxoplasmic lymphadenopathy. $J$ Clin Pathol. 2005; 58(11):1143-6. http://dx.doi.org/10.1136/jcp.2005.026492

13. Bilal JA, Alsammani MA, Ahmed MI. Acute Toxoplasma gondii infection in children with reactive hyperplasia of the cervical lymph nodes. Saudi Med J. 2014; 35(7):699-703. No doi

14. Paul M. Immunoglobulin $\mathrm{G}$ avidity in diagnosis of toxoplasmic lymphadenopathy and ocular toxoplasmosis. Clin Diagn Lab Immunol. 1999; 6(4):514-8. No doi

15. Lin MH, Chen TC, Kuo TT, et al. Real-time PCR for quantitative detection of Toxoplasma gondii. J Clin Microbiol. 2000; 38:4121-5. No doi

16. Stansfeld AG. The histological diagnosis of toxoplasmic lymphadenitis. J Clin Pathol. 1961; 14(6):565-573. No doi

17. Montoya JG, Remington JS. Studies on the sero-diagnosis of toxoplasmic lymphadenitis. Clin Infect Dis. 1995; 20(4):781-9. http://dx.doi.org/10.1093/clinids/20.4.781 